\title{
Rede de correlações entre qualidade de vida, resiliência e desequilíbrio esforço-recompensa em policiais militares
}

\author{
Network of correlations between quality of life, resilience and \\ effort-reward imbalance in military police officers
}

Juliana Petri Tavares (https://orcid.org/0000-0003-4121-645X) ${ }^{1}$

Lizandra Santos Vieira (https://orcid.org/0000-0002-4303-7079) ${ }^{2}$

Daiane Dal Pai (https://orcid.org/0000-0002-6761-0415) ${ }^{2}$

Sônia Beatriz Cócaro de Souza (https://orcid.org/0000-0002-6980-1098) ${ }^{1}$

Roger Flores Ceccon (https://orcid.org/0000-0002-0846-1376) ${ }^{3}$

Wagner de Lara Machado (https://orcid.org/0000-0001-5555-5116) ${ }^{4}$

${ }^{1}$ Departamento de Enfermagem MédicoCirúrgica, Escola de Enfermagem, Universidade Federal do Rio Grande do Sul. R. São Manoel, 963, Rio Branco. 90620-110. Porto Alegre RS Brasil. jupetritavares@gmail.com ${ }^{2}$ Programa de Pós-

Graduação em Enfermagem Escola de Enfermagem,

Universidade Federal do Rio Grande do Sul. Porto Alegre RS Brasil.

${ }^{3}$ Universidade Federal de Santa Catarina. Araranguá SC Brasil.

${ }^{4}$ Escola de Ciências

da Saúde, Pontifícia

Universidade Católica do

Rio Grande do Sul. Porto

Alegre RS Brasil.

\begin{abstract}
Objective: To analyze the network of correlations between the realms of the Effor $t$-Reward Imbalance (ERI) model, resilience, and quality of life among military police officers. Method: This is a cross-sectional study conducted with 258 police officers from the Special Opera tions Battalion of the Military Police in a city in Rio Grande do Sul, Brazil. The scales of the ERI model, resilience, and World Health Quality of Life (WHOQOL-bref) assessed psychosocial stress, resilience, and quality of life. Descriptive and analytical statistics were performed em ploying the network analysis. Results: The realms of the ERI model and resilience correlated with all the WHOQOL's realms $(p<0.001)$. The network model showed a negative association between effort, overcommitment, and physical and environmental realms. The environmental realm was positively associated with reward, while resilience was positively associated with the general, physical, and psychological realms. Conclusion: We concluded that psychosocial stress interferes in the quality of life of military police officers, and resi lience may work as a protective factor.
\end{abstract}

Key words Occupational health, Police, Resilience, Psychological, Stress, Psychological, Quality of life
Resumo Objetivo: Analisar a rede de correlações entre as dimensões do Modelo Desequilíbrio Esforço-Recompensa (DER), resiliência e qualidade de vida em policiais militares. Método: Estudo transversal realizado com 258 policiais do Batalhão de Operações Especiais (BOE) da Polícia Militar de uma cidade do Rio Grande do Sul. As escalas do Modelo DER, de resiliência e World Health Quality of Life (WHOQOL-bref) avaliaram o estresse psicossocial, resiliência e qualidade de vida. Foram realizadas estatísticas descritiva e analítica, com análise de rede. Resultados: As dimensões do Modelo DER e a resiliência se correlacionaram com todos os dominios do WHOQOL $(p<0,001)$. O modelo de rede indicou associação negativa entre o esforço, o excesso de comprometimento e os domínios fisico e meio ambiente. O dominio meio ambiente foi associado positivamente à recompensa, e a resiliência associou-se positivamente com os domínios geral, físico e psicológico. Conclusão: Concluiu-se que o estresse psicossocial interfere na qualidade de vida dos policiais militares e a resiliência pode atuar como um fator de proteção.

Palavras-chave Saúde do trabalhador, Polícia, Resiliência psicológica, Estresse psicológico, Qualidade de vida 


\section{Introdução}

O trabalho policial, em todos os países do mundo é caracterizado pela exposição ao estresse e risco de morte ${ }^{1-3}$, principalmente entre policiais militares. O nível de estresse é superior ao de outras categorias profissionais devido às características das atividades realizadas, à sobrecarga de trabalho, às relações interpessoais, à baixa remuneração ${ }^{4}$, e às precárias condições de trabalho ${ }^{5}$.

Congregado a estas condições, a violência urbana é um dos grandes problemas sociais de países pobres e desiguais ${ }^{6}$. No Brasil, há forte relação entre espaço geográfico e criminalidade, visto que nas regiões onde há maior taxa de urbanização e densidade demográfica há maior prevalência de violências ${ }^{7}$. Além do mais, as precárias condições de trabalho e a responsabilidade com a segurança pública em um contexto de grande criminalidade no país demonstram um alto empenho exercido e baixas recompensas, que podem levar à situação de estresse psicossocial.

A avaliação do estresse psicossocial pode ser realizada pelo Modelo Desequilíbrio Esforço-Recompensa (DER), desenvolvido para identificar efeitos adversos à saúde ante a estressores laborais e condições precárias no mundo do trabalho em países desenvolvidos e em rápido desenvolvimento. Postula que a falta de reciprocidade no trabalho, caracterizado pelo "alto custo/baixo ganho", ou seja, a frustração de recompensas após esforços apropriados, aumenta o risco de transtornos relacionados ao estresse ${ }^{8}$.

O estresse psicossocial pode estar associado a fatores organizacionais do trabalho e a aspectos individuais e subjetivos dos profissionais, especialmente a resiliência, caracterizada pela adaptação criativa e individual frente aos riscos e adversidades do cotidiano do trabalho ${ }^{9}$. Dessa forma, a resiliência é importante para a saúde mental dos trabalhadores, e se constitui como um fator de proteção contra o estresse laboral ${ }^{9}$ e um dispositivo de melhoria da qualidade de vida.

O conceito de qualidade de vida é subjetivo, multidimensional e inclui elementos positivos e negativos. É caracterizado pela percepção do indivíduo de sua posição na vida, no contexto da cultura, nos sistemas de valores nos quais vive e na relação com seus objetivos, expectativas, padrões e preocupações ${ }^{10}$. É um conceito amplo e complexo, que engloba a saúde física, estado psicológico, nível de independência, relações sociais, crenças pessoais e relação com as características do meio ambiente ${ }^{11}$.

Ao longo dos últimos anos foram realizados poucos estudos no Brasil e no mundo sobre re- siliência em policiais ${ }^{12}$, alterações psíquicas e sua relação com qualidade de vida ${ }^{2,13}$. Assim, a relação entre as dimensões do Modelo DER, resiliência e qualidade de vida em policiais militares permanece como lacuna na produção do conhecimento.

Nesta perspectiva, a polícia militar representa um coletivo vulnerável de trabalhadores, que sente o impacto negativo da atividade laboral na saúde e na qualidade de vida. Este efeito é agravado pelos acréscimos permanentes na responsabilidade de zelar pela segurança da população, em contraponto do aumento da criminalidade e diminuição dos investimentos na segurança pública nos últimos anos no Brasil. Este desnível entre a alta demanda dos profissionais, associado às baixas recompensas, podem ser fatores de risco para o desenvolvimento de estresse psicossocial e prejuízos à qualidade de vida. Ante o exposto, este estudo tem como objetivo analisar a rede de correlações entre as dimensões do Modelo DER, resiliência e qualidade de vida em policiais militares.

\section{Método}

Estudo transversal realizado no Batalhão de Operações Especiais (BOE) da Polícia Militar de uma cidade de grande porte populacional do estado do Rio Grande do Sul. A população do estudo era composta de 416 policiais. Foram incluídos na amostra os sujeitos ativos, na faixa etária entre 18 e 65 anos, lotados no BOE. Estabeleceram-se como critérios de exclusão da amostra os policiais afastados da sua função ou em licença por qualquer motivo no período de coleta dos dados, os com tempo de trabalho inferior a um ano na corporação, e os do sexo feminino, por ser inferior a $5 \%$ da amostra. Com base nesses critérios, a população elegível do estudo foi de 263 policiais militares. Todos os sujeitos elegíveis foram abordados. Destes, 258 responderam o instrumento de coleta de dados.

Sobre os dados, referiu-se a coletas de informações relativas às características sociolaborais, estresse psicossocial e resiliência, no ano de 2013, na sede do BOE.

Os dados sociolaborais compreenderam a idade, situação conjugal, posto/graduação, tempo de trabalho na função, trabalho em outro lugar, horas extras, turno de trabalho, tabagismo, horas de sono, problemas de saúde, lazer, medidas antropométricas e cardiovasculares (pressão arterial, peso, altura, índice de massa corporal, circunferência abdominal, circunferência do quadril). 
O estresse psicossocial foi mensurado por meio da escala do Modelo Desequilíbrio EsforçoRecompensa (DER), versão curta $^{14}$, validada no Brasil $^{15}$ e considerada confiável $(\alpha=0,703)$. Realizou-se, neste estudo, a análise fatorial da escala, na qual as questões ficaram agrupadas em três fatores: recompensa, esforço e excesso de comprometimento. Os dados das três dimensões foram analisados de modo contínuos.

Para avaliar a resiliência usou-se o instrumento desenvolvido por Wagnild e Young ${ }^{16}$, validado no Brasil ${ }^{17}$. O instrumento World Health Quality of Life (WHOQOL-bref) foi utilizado para avaliação da qualidade de vida, desenvolvido pela Organização Mundial da Saúde e adaptado e validado no Brasil ${ }^{18}$. O WHOQOL-bref possui 26 questões agrupadas em cinco domínios: físico (DF), psicológico (DP), relações sociais (DRS), meio ambiente (DMA) e geral (DG).

Os dados coletados foram organizados em planilhas no programa Excel $^{\circledR}$. Em seguida, realizou-se a transposição deles no programa SPSS ${ }^{\circledR}$ (Statistical Package for the Social Sciences, SPSS Inc, Chicago) versão 18.0 para Windows. Por conseguinte, analisados por meio de técnicas de estatística descritiva e inferencial. As variáveis categóricas foram apresentadas com frequências absolutas e relativas. As variáveis contínuas, apresentadas como medidas de tendência central (média e mediana) e de dispersão (desvio-padrão e intervalos quartis). Realizou-se o teste de normalidade de Shapiro-Wilk para verificar a distribuição das variáveis, além dos valores de assimetria e curtose. O teste $t$ de Student foi utilizado para associação entre variáveis com distribuição simétrica, enquanto que para assimétrica utilizou-se o Mann-Whitney. Foram realizadas correlações bivariadas de Pearson (para variáveis simétricas) e de Spearman (para variáveis assimétricas) para avaliar a relação entre variáveis contínuas e o desfecho qualidade de vida.

Para investigar as relações entre os domínios de qualidade de vida, a resiliência e as dimensões do Modelo DER, foi conduzida uma análise de rede que compreende duas etapas: a primeira consiste em estimar a matriz de correlações parciais regularizadas; a segunda representa a matriz de correlações parciais em um plano bidimensional em um objeto gráfico. Vértices representam as variáveis investigadas e arestas representam a relação parcial entre elas, podendo variar em espessura (magnitude) e padrão (positiva ou negativa). Um algoritmo de posicionamento é utilizado, no qual aproxima/afasta as variáveis em função de sua associação ${ }^{19}$. Medidas descritivas da rede também foram reportadas, o que indica o nível de associação e influência esperada de cada variável.

Por fim, foram conduzidas análises de comunidades para identificar subgrupos presentes dentro do sistema. $\mathrm{O}$ algoritmo utilizado foi um método hierárquico ${ }^{20}$, o qual se baseia na otimização da medida de modularidade (associação intravértices), partindo do modelo mais simples, de um agrupamento, seguindo para a divisão do grafo em $\mathrm{n}$ subgrupos. O número de agrupamentos é definido pelo menor valor da modularidade. Esta análise teve por objetivo identificar agrupamentos de preditores no sistema investigado.

A pesquisa foi aprovada pelo Comitê de Ética em Pesquisa da Universidade Federal do Rio Grande do Sul. Foram respeitados os princípios éticos e todos os sujeitos que participaram da pesquisa assinaram o Termo de Consentimento Livre e Esclarecido.

\section{Resultados}

Os policiais militares apresentaram mediana de 36 anos de idade (31-45), 11 anos de estudo (1113) e 1 filho (0-2). O maior percentual possuía companheiro $(69,0 \%)$, era soldado $(75,1 \%)$, possuía outro emprego $(61,6 \%)$ e realizava horas extras $(67,4 \%)$. Quando avaliado o estilo de vida, os policiais apresentaram mediana de 6 horas de sono diárias (6-7), 89,5\% não era tabagista, 64\% possuíam pouco tempo para lazer, $23,8 \%$ apresentavam problemas de saúde e $15,5 \%$ faziam uso de medicações.

Com relação às características antropométricas e cardiovasculares dos policiais, a mediana da pressão sistólica foi $134 \mathrm{mmHg}(125 ; 145)$; pressão diastólica $86 \mathrm{mmHg}(77,75 ; 94,0)$; circunferência abdominal $91,5 \mathrm{~cm}(85,75 ; 97)$; e circunferência do quadril $103 \mathrm{~cm}(99 ; 108)$. O peso e a altura apresentaram as médias $84,6 \pm 10,6 \mathrm{~kg}$ e $176,6 \pm 6,2,4 \mathrm{~cm}$, respectivamente.

Os policiais que não apresentavam problemas de saúde, que não faziam uso de medicamentos e que não trabalhavam em outro emprego apresentaram maiores médias no domínio físico (DF) do WHOQOL-breve $(p<0,05)$. Houve correlação entre as variáveis peso $(r=-0,148 ; p=0,020)$, circunferência abdominal $(\mathrm{r}=-0,193 ; \mathrm{p}=0,002)$ variável hora de sono $(\mathrm{r}=0,180 ; \mathrm{p}=0,005)$ e o $\mathrm{DF}$. O domínio psicológico (DP) apresentou maiores médias para os polìciais não fumantes, sem companheiro, que não apresentavam problemas de saúde e não possuíam outro emprego $(\mathrm{p}<0,05)$. 
Os policiais com problemas de saúde e com outro trabalho apresentaram menores médias no domínio das relações sociais (DRS) $(\mathrm{p}<0,05)$. O mesmo domínio se correlacionou inversamente com a circunferência abdominal $(r=-0,150$; $\mathrm{p}=0,018)$. O domínio meio ambiente (DMA) apresentou maiores médias para a categoria trabalho diurno $(\mathrm{p}=0,037)$ e correlacionou-se com a satisfação com a remuneração $(r=0,325$; $\mathrm{p}<0,001)$.

O domínio geral (DG) do WHOQOL apresentou maiores médias para os policiais não tabagistas, que não possuíam problemas de saúde, não faziam uso de medicações e que trabalhavam no turno diurno. Adicionalmente, apresentou correlação com circunferência abdominal ( $\mathrm{r}=-$ $0,143 ; 0,021)$, pressão arterial sistólica $(\mathrm{r}=0,136$; $\mathrm{p}=0,039)$ e horas de sono $(\mathrm{r}=0,203 ; \mathrm{p}=0,001)$.

As dimensões recompensa (R) e esforço (E) do Modelo DER, o excesso de comprometimento (EC) e a resiliência (Res) correlacionaram-se com todos os domínios do WHOQOL ( $\mathrm{p}<0,001$ ), conforme Tabela 1.
A rede de correlações parciais regularizadas entre os domínios de qualidade de vida, resiliência e as dimensões do Modelo DER é representada na Figura 1.

As arestas indicam associações positivas entre os domínios de qualidade de vida, resiliência e dimensão de "recompensa" do Modelo DER (linhas contínuas), e associações negativas entre os domínios físico e meio ambiente e as dimensões "esforço" do Modelo DER e "excesso de comprometimento". Nesta figura é possível perceber também os subagrupamentos identificados pelo algoritmo de Louvain. Este resultado indica que, diferentemente dos domínios da qualidade de vida, o domínio "meio ambiente" está mais associado à dimensão "recompensa" do Modelo DER. As demais variáveis possuem associações maiores com seus próprios agrupamentos.

Analisando as medidas de centralidade da rede de correlações parciais regularizadas (Figura 2) é possível identificar variáveis mais relevantes neste sistema, possíveis candidatas à intervenção, no sentido de produzir mudanças nas demais

Tabela 1. Distribuição dos domínios da qualidade de vida (WHOQOL-bref), segundo variáveis laborais e de estilo de vida. Porto Alegre, RS, Brasil, 2013 ( $\mathrm{n}=258$ ).

\begin{tabular}{|c|c|c|c|c|c|}
\hline Variáveis & DF & DP & DRS & DMA & DG \\
\hline \multicolumn{6}{|l|}{ Situação conjugal } \\
\hline Companheiro & $15,11 \pm 2,21$ & $15,42 \pm 2,12$ & $15,92 \pm 2,45$ & $13,20 \pm 2,47$ & $15,42 \pm 2,13$ \\
\hline \multirow[t]{2}{*}{ Sem companheiro } & $15,62 \pm 2,14$ & $16,06 \pm 1,97$ & $16,25 \pm 2,54$ & $13,58 \pm 2,26$ & $16,06 \pm 2,60$ \\
\hline & $\mathrm{p}=0,094$ & $\mathrm{p}=\mathbf{0 , 0 2 8}$ & $\mathrm{p}=0,345$ & $\mathrm{p}=0,240$ & $\mathrm{p}=0,163$ \\
\hline \multicolumn{6}{|l|}{ Tabagista } \\
\hline Não & $15,53 \pm 2,17$ & $15,98 \pm 1,98$ & $16,25 \pm 2,48$ & $13,53 \pm 2,28$ & $15,60 \pm 2,40$ \\
\hline \multirow[t]{2}{*}{ Sim } & $14,92 \pm 2,02$ & $14,74 \pm 2,11$ & $15,46 \pm 2,66$ & $12,86 \pm 2,64$ & $14,44 \pm 2,85$ \\
\hline & $\mathrm{p}=0,183$ & $p=0,004$ & $\mathrm{p}=0,136$ & $\mathrm{p}=0,170$ & $\mathrm{p}=0,021$ \\
\hline \multicolumn{6}{|l|}{ Problemas de saúde } \\
\hline Não & $15,75 \pm 2,09$ & $16,06 \pm 2,10$ & $16,38 \pm 2,42$ & $13,64 \pm 2,25$ & $15,64 \pm 2,48$ \\
\hline \multirow[t]{2}{*}{ Sim } & $14,54 \pm 2,17$ & $15,21 \pm 2,0$ & $15,48 \pm 2,62$ & $13,25 \pm 2,53$ & $14,91 \pm 2,35$ \\
\hline & $\mathrm{p}<\mathbf{0 , 0 0 1}$ & $p=0,006$ & $p=0,016$ & $\mathrm{p}=0,436$ & $p=0,046$ \\
\hline \multicolumn{6}{|l|}{ Medicações } \\
\hline Não & $15,64 \pm 2,09$ & $15,95 \pm 2,02$ & $16,29 \pm 2,41$ & $13,54 \pm 2,28$ & $15,95 \pm 2,02$ \\
\hline \multirow[t]{2}{*}{ Sim } & $14,51 \pm 2,31$ & $15,33 \pm 2,04$ & $15,49 \pm 2,97$ & $12,98 \pm 2,53$ & $15,33 \pm 2,04$ \\
\hline & $\mathrm{p}=\mathbf{0 , 0 0 3}$ & $\mathrm{p}=0,085$ & $\mathrm{p}=0,073$ & $\mathrm{p}=0,171$ & $p=0,021$ \\
\hline \multicolumn{6}{|l|}{ Trabalho outro } \\
\hline Não & $15,71 \pm 2,15$ & $16,10 \pm 1,97$ & $16,45 \pm 2,41$ & $13,56 \pm 2,26$ & $15,58 \pm 2,38$ \\
\hline \multirow[t]{2}{*}{ Sim } & $15,07 \pm 2,15$ & $15,46 \pm 2,07$ & $15,68 \pm 2,64$ & $13,26 \pm 2,43$ & $15,30 \pm 2,64$ \\
\hline & $p=0,027$ & $p=0,018$ & $\mathrm{p}=\mathbf{0 , 0 2 2}$ & $\mathrm{p}=0,340$ & $\mathrm{p}=0,373$ \\
\hline \multicolumn{6}{|l|}{ Turno de trabalho } \\
\hline Diurno & $15,51 \pm 2,27$ & $16,13 \pm 2,04$ & $16,56 \pm 2,35$ & $14,01 \pm 2,10$ & $15,94 \pm 2,33$ \\
\hline \multirow[t]{2}{*}{ Diurno e noturno } & $15,47 \pm 2,06$ & $15,65 \pm 2,06$ & $15,88 \pm 2,73$ & $13,16 \pm 2,43$ & $15,27 \pm 2,42$ \\
\hline & $\mathrm{p}=0,892$ & $\mathrm{p}=0,088$ & $\mathrm{p}=0,052$ & $\mathrm{p}=\mathbf{0 , 0 0 7}$ & $p=0,037$ \\
\hline \multirow[t]{2}{*}{ Idade } & $-0,072$ & 0,030 & $-0,096$ & 0,070 & 0,014 \\
\hline & $\mathrm{p}=0,260$ & $\mathrm{p}=0,643$ & $\mathrm{p}=0,137$ & $\mathrm{p}=0,276$ & $\mathrm{p}=0,828$ \\
\hline
\end{tabular}


Tabela 1. Distribuição dos domínios da qualidade de vida (WHOQOL-bref), segundo variáveis laborais e de estilo de vida. Porto Alegre, RS, Brasil, 2013 ( $\mathrm{n}=258$ ).

\begin{tabular}{|c|c|c|c|c|c|}
\hline Variáveis & DF & DP & DRS & DMA & DG \\
\hline \multirow[t]{2}{*}{ No de filhos } & $-0,056$ & $-0,042$ & $-0,092$ & $-0,028$ & $-0,077$ \\
\hline & $\mathrm{p}=0,388$ & 0,511 & $\mathrm{p}=0,153$ & $\mathrm{p}=0,666$ & $\mathrm{p}=0,221$ \\
\hline \multirow[t]{2}{*}{ Escolaridade } & 0,022 & $-0,042$ & $-0,016$ & $-0,042$ & $-0,036$ \\
\hline & $\mathrm{p}=0,734$ & $\mathrm{p}=0,523$ & $\mathrm{p}=0,803$ & $\mathrm{p}=0,523$ & $\mathrm{p}=0,576$ \\
\hline \multirow[t]{2}{*}{ Peso } & $-0,148$ & 0,004 & $-0,029$ & 0,004 & $-0,073$ \\
\hline & $\mathrm{p}=\mathbf{0 , 0 2 0}$ & $\mathrm{p}=0,946$ & $\mathrm{p}=0,648$ & $\mathrm{p}=0,946$ & $\mathrm{p}=0,245$ \\
\hline \multirow[t]{2}{*}{ Altura } & $-0,068$ & $-0,068$ & $-0,068$ & $-0,068$ & $-0,055$ \\
\hline & $\mathrm{p}=0,284$ & $\mathrm{p}=0,288$ & $\mathrm{p}=0,165$ & $\mathrm{p}=0,288$ & 0,382 \\
\hline \multirow[t]{2}{*}{ Circunferência abdominal } & $-0,193$ & 0,014 & $-0,150$ & 0,014 & $-0,143$ \\
\hline & $p=0,002$ & $\mathrm{p}=0,830$ & $p=0,018$ & $\mathrm{p}=0,830$ & 0,021 \\
\hline \multirow[t]{2}{*}{ Circunferência quadril } & $-0,116$ & 0,072 & $-0,049$ & 0,072 & $-0,096$ \\
\hline & $\mathrm{p}=0,069$ & $\mathrm{p}=0,259$ & $\mathrm{p}=446$ & $\mathrm{p}=0,259$ & $\mathrm{p}=0,125$ \\
\hline \multirow[t]{2}{*}{ Pressão arterial sistólica } & 0,030 & 0,118 & 0,010 & 0,118 & 0,136 \\
\hline & $\mathrm{p}=0,653$ & $\mathrm{p}=0,082$ & $\mathrm{p}=0,881$ & $\mathrm{p}=0,082$ & $\mathrm{p}=\mathbf{0 , 0 3 9}$ \\
\hline \multirow[t]{2}{*}{ Pressão arterial diastólica } & $-0,035$ & 0,102 & $-0,013$ & 0,102 & $-0,009$ \\
\hline & $\mathrm{p}=0,606$ & $\mathrm{p}=0,132$ & $\mathrm{p}=0,845$ & $\mathrm{p}=0,132$ & $\mathrm{p}=0,893$ \\
\hline \multirow[t]{2}{*}{ Satisfação remuneração } & 0,118 & 0,109 & 0,075 & 0,325 & 0,102 \\
\hline & $\mathrm{p}=0,065$ & $\mathrm{p}=0,089$ & $\mathrm{p}=0,241$ & $\mathrm{p}<\mathbf{0 , 0 0 1}$ & $\mathrm{p}=0,104$ \\
\hline \multirow[t]{2}{*}{ Tempo de serviço } & $-0,060$ & 0,022 & $-0,068$ & 0,092 & 0,036 \\
\hline & $\mathrm{p}=0,347$ & $\mathrm{p}=0,734$ & $\mathrm{p}=0,283$ & $\mathrm{p}=0,150$ & $\mathrm{p}=0,570$ \\
\hline \multirow[t]{2}{*}{ Tempo na função } & $-0,117$ & 0,019 & $-0,042$ & 0,022 & $-0,009$ \\
\hline & $\mathrm{p}=0,067$ & $\mathrm{p}=0,766$ & $\mathrm{p}=0,509$ & 0,729 & 0,881 \\
\hline \multirow[t]{2}{*}{ Horas de sono } & 0,180 & 0,119 & 0,078 & 0,074 & 0,203 \\
\hline & $\mathrm{p}=0,005$ & $\mathrm{p}=0,064$ & $\mathrm{p}=0,226$ & $\mathrm{p}=0,247$ & $\mathrm{p}=0,001$ \\
\hline \multirow[t]{2}{*}{ Resiliência (RES) } & 0,437 & 0,501 & 0,366 & 0,354 & 0,420 \\
\hline & $\mathrm{p}<0,001$ & $\mathrm{p}<0,001$ & $\mathrm{p}<0,001$ & $\mathrm{p}<0,001$ & $\mathrm{p}<\mathbf{0 , 0 0 1}$ \\
\hline \multirow[t]{2}{*}{ Esforço (E) } & $-0,412$ & $-0,352$ & $-0,257$ & $-0,324$ & $-0,266$ \\
\hline & $\mathrm{p}<0,001$ & $\mathrm{p}<0,001$ & $\mathrm{p}<\mathbf{0 , 0 0 1}$ & $\mathrm{p}<0,001$ & $\mathrm{p}<0,001$ \\
\hline \multirow[t]{2}{*}{ Recompensa (R) } & 0,208 & 0,252 & 0,197 & 0,350 & 0,169 \\
\hline & $\mathrm{p}=0,001$ & $\mathrm{p}<0,001$ & $p=0,02$ & $\mathrm{p}<\mathbf{0 , 0 0 1}$ & $\mathrm{p}=\mathbf{0 , 0 0 7}$ \\
\hline \multirow{2}{*}{$\begin{array}{l}\text { Excesso de comprometimento } \\
\text { (EC) }\end{array}$} & $-0,486$ & $-0,430$ & $-0,306$ & $-0,354$ & $-0,292$ \\
\hline & $\mathrm{p}<\mathbf{0 , 0 0 1}$ & $\mathrm{p}<0,001$ & $\mathrm{p}<\mathbf{0 , 0 0 1}$ & $\mathrm{p}<0,001$ & $\mathrm{p}<\mathbf{0 , 0 0 1}$ \\
\hline
\end{tabular}

Fonte: Dados da pesquisa, 2013.

variáveis. A medida de proximidade indica o domínio físico da qualidade de vida como variável mais associada às demais variáveis no sistema. Isto indica que ela pode ser uma variável importante para transmitir efeitos do estresse nos demais domínios da qualidade de vida. Sendo assim, ela é a variável que mais se relaciona aos fatores de risco, e por isso, acaba por influenciar negativamente a percepção de qualidade de vida em outros domínios. Já a medida de influência esperada indica as variáveis que, uma vez ativadas, possuem uma maior probabilidade de gerar variações (positivas ou negativas) nas variáveis próximas. Os domínios geral e psicológico da qualidade de vida, uma vez modificados, tendem a produzir variações positivas nas variáveis próximas, no caso, os demais domínios de qualidade de vida. Já a variável excesso de comprometimento, uma vez ativada, tende a produzir variações negativas nas variáveis próximas, no caso, os domínios de qualidade de vida, especialmente físico e meio ambiente.

\section{Discussão}

Os resultados apresentados neste artigo despertam reflexões sobre os fatores que podem interferir na qualidade de vida dos policiais militares, dentre eles características pessoais, laborais, es- 


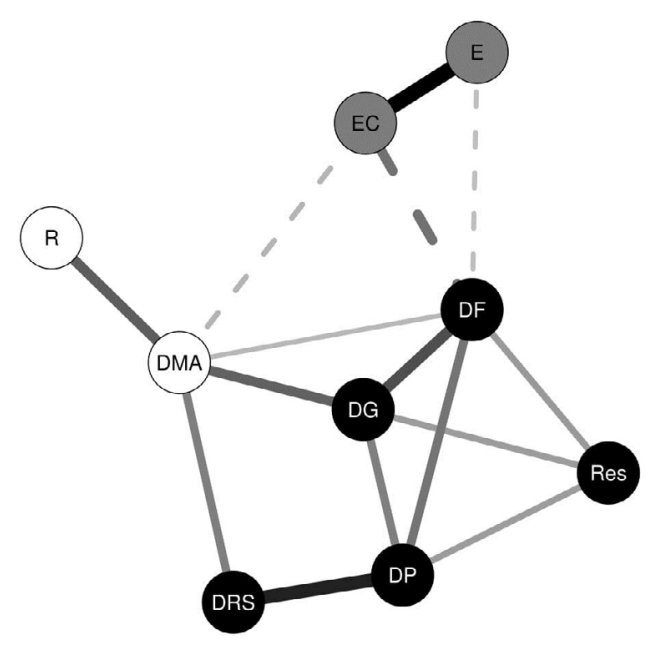

Figura 1. Rede de correlações parciais regularizadas entre os domínios de qualidade de vida (DF, DP, DMA, DRS e DG), resiliência (R) e dimensões do Modelo DER (E, Res e EC). Vértices representam variáveis e arestas representam associações positivas (traço contínuo) e negativas (traço pontilhado). A cor do vértices representam comunidades (subgrupos) identificados na análise de comunidades.

tresse psicossocial e resiliência. Algumas características individuais como problemas de saúde, uso de medicamentos, horas de sono, consumo de tabaco, circunferência abdominal, bem como variáveis laborais (possuir outro emprego, satisfação com a remuneração e turno de trabalho) repercutiram na qualidade de vida dos policiais militares deste estudo.

As médias mais altas do domínio físico (DF) se relacionaram com a ausência de um segundo emprego e inversamente ao peso, à circunferência abdominal e ao sono, logo sugere que uma carga de trabalho maior interfere na qualidade de vida dos policiais e, consequentemente, em menor tempo para descanso, atividades físicas e cuidados com a saúde. Estudo realizado no estado de Minas Gerais identificou que a menor percepção dos policiais sobre o domínio físico está associada a características da sua atividade laboral militar, as mudanças de turnos de trabalho, o tipo de atividade a qual o militar é submetido e a relação das jornadas de trabalho e repouso interferem drasticamente em parâmetros ligados a qualidade de vida como o sono e a manutenção da atividade física regular ${ }^{21}$.

Os achados mostraram que além de interferir no domínio físico, possuir outro emprego impacta no domínio das relações sociais e no domínio psicológico da qualidade de vida. Em consonância, estudo aponta que o convívio familiar e apoio social dos amigos são parâmetros indispensáveis para a qualidade de vida de policiais militares ${ }^{21}$, e podem ser prejudicados em decorrência do excesso de trabalho e acúmulo de funções dos policiais ao exercerem mais de um atividade.

O uso de tabaco somado à carência de hábitos de vida saudáveis impacta sobre a saúde. Estudo realizado na Etiópia mostrou que $88,2 \%$ dos policiais não possuíam alimentação saudável, que $70 \%$ consumiam álcool e que $36,1 \%$ eram inativos quanto à atividade física. Além disso, 6,7\% eram tabagistas, sendo $83 \%$ fumantes diários, o que aponta prejuízos à qualidade de vida ${ }^{22}$. $\mathrm{O}$ domínio psicológico apresentou menores médias para os policiais tabagistas, podendo estar relacionado tanto às consequências quanto aos fatores que levam ao uso do tabaco. Além disso, policiais com problemas de saúde apresentaram menores médias no domínio das relações sociais.

Visto que o trabalho do policial militar requer uso intenso e frequente da força física, as variáveis antropométricas apresentaram valores limítrofes de referência, o que indica a necessidade de atenção para a saúde dos policiais, e demonstra relação com a menor qualidade de vida, menos domínios psicológico e físico, e problema nas relações sociais. Outro estudo brasileiro e que visa identificar o nível de atividade física dos policiais militares, mostrou que estavam fora da normalidade populacional no que se refere ao percentual de gordura, relação circunferência cintura-quadril, índice de massa corporal e inativos segundo o Questionário Internacional de Atividade Física, apresentando alto ou moderado risco à saúde ${ }^{23}$. No presente estudo, o domínio geral (DG) apresentou maiores médias para os policiais não tabagistas, que não têm problemas de saúde e que não fazem uso de medicamentos, o que apresenta relação com circunferência abdominal e pressão arterial sistólica.

O domínio meio ambiente (DMA) foi maior para os policiais que trabalhavam no turno diurno e que estavam mais satisfeitos com a renda mensal. Segundo estudo ${ }^{21}$, em relação ao domínio meio ambiente, a baixa percepção dos militares está associada ao fato de que todos os indivíduos avaliados estão lotados em companhias 

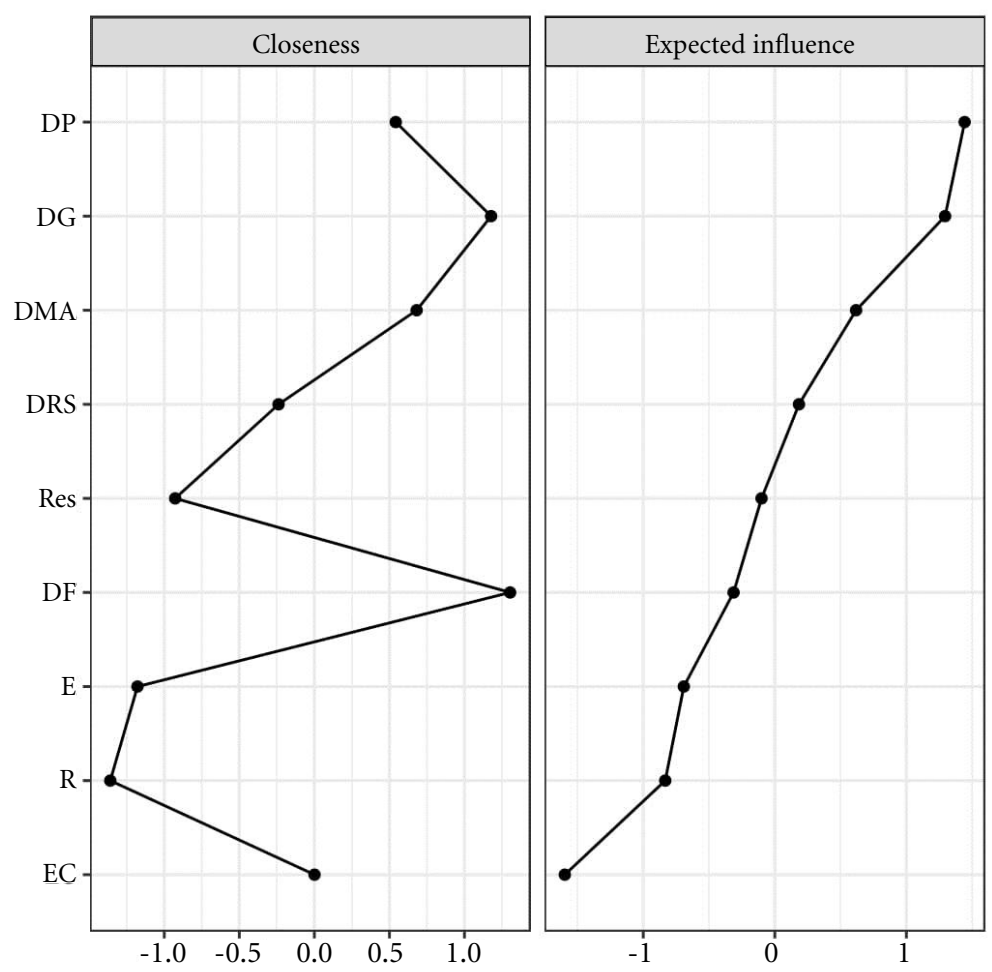

Figura 2. Medidas padronizadas de proximidade (closeness) e de influência esperada (expected influence) para a rede de correlações parciais regularizadas. A medida de closeness indica as variáveis mais associadas às demais variáveis no sistema (altos valores positivos). A medida de influência esperada indica as variáveis que desativam (altos valores negativos) e ativam (altos valores positivos) outras variáveis no sistema.

metropolitanas de polícia. Fatores estressantes presentes em grandes metrópoles como o tempo gasto no transporte, a falta de segurança física e a proteção para os militares e seus familiares, bem como o custo de vida, fazem com que a percepção sobre a qualidade de vida seja menor. Outro estudo apontou o comprometimento da qualidade de vida dos policiais militares do interior do estado de São Paulo nos fatores relacionados ao domínio meio ambiente, o que sugere a necessidade de melhorias para questões voltadas para a segurança física e proteção desses profissionais, as condições ambientais do local onde estão inseridos, os recursos financeiros e de transporte, além do ambiente no lar $^{24}$.

No Brasil, além dos fatores de risco inerentes à profissão do policial militar, acrescenta-se a falta de estrutura para o desenvolvimento do trabalho, o que inclui a necessidade de apoio psicológico aos trabalhadores. Estudo realizado no Rio Grande do Sul (Brasil) apontou que há insa- tisfação entre policiais em relação ao trabalho e, quanto às dimensões que compõem esse modelo teórico, registrou-se insatisfação em relação ao salário e a promoções na carreira ${ }^{25}$.

Os policiais civis e militares que atuavam em Unidades de Operações Especiais no estado de Santa Catarina apresentaram percepção regular de suas condições de trabalho. Os componentes remuneração, benefícios e ambiente fisico foram percebidos de forma mais negativa. Em relação ao estresse ocupacional, a maioria dos policiais considerou seu trabalho como de baixa demanda, controle e apoio social. Além disso, observou-se relação direta, de forma inversa, entre as condições de trabalho e o estresse ocupacional ${ }^{5}$.

Estudo realizado na Grécia, com o objetivo de examinar o estresse percebido, satisfação no trabalho, qualidade de vida e suas relações, mostraram que os níveis de estresse percebidos têm um efeito negativo importante com relação à satisfação no trabalho e na qualidade de vida. Os 
oficiais de maior escalão tiveram maior nível de insatisfação com seu trabalho².

Evidenciou-se neste estudo que o domínio geral (DG), o domínio psicológico (DP) e a resiliência podem atuar como fatores de proteção em relação ao estresse e à percepção negativa de outros domínios de qualidade de vida. A resiliência psicológica tem uma capacidade de evitar distúrbios de saúde mental em médio prazo, especificamente quando estes estão em fases iniciais $^{12}$. Estudo aponta que, embora os governos de países desenvolvidos da Europa, Canadá e Estados Unidos tenham investido em treinamento tático e equipamentos tecnológicos, a resiliência não tem sido um componente da formação policial $^{26}$. Acrescenta ainda que é fundamental fortalecer ferramentas para promover a resiliência e manter o bem estar pessoal, visto que os policiais são rotineiramente encarregados de enfrentar a vida em risco ${ }^{26}$.

A resiliência, como um fator de proteção, pode eventualmente ajudar a identificar indivíduos - como policiais, bombeiros e policiais militares - que estão com alto risco de desenvolver doenças relacionadas ao trauma ${ }^{27}$. Esta grande exposição a fatores de risco, inerentes à profissão, reflete na menor qualidade de vida com a presença de depressão, doença física e altos níveis de estresse $^{28}$.

Estudo realizado na Índia destacou que a maioria das fontes percebidas de estresse no trabalho está relacionada a problemas no local de trabalho, podendo ser realizadas mudanças dentro do departamento de polícia para prevenir o estresse e seus efeitos. Entre as medidas estão o treinamento adequado para aliviar o estresse e aumentar a autoeficácia e habilidades de enfrentamento, e os programas departamentais de saúde mental para tratar problemas relacionados ao estresse. A instituição policial também pode inserir passos para a melhora da imagem pública da polícia ${ }^{29}$.

Programas de prevenção devem ser oferecidos aos policiais, com atividades pró-ativas dos trabalhadores relacionadas à análise crítica do estresse, investindo em preparações mentais empiricamente testadas e baseadas em evidências ${ }^{26}$. Ademais, a recompensa também exerce função fundamental na qualidade de vida do policial, e é fruto da valorização e condições adequadas de trabalho.

As limitações do estudo referem-se à carência de artigos sobre resiliência, estresse e qualidade de vida em policiais, o que permitiria avançar na discussão a respeito de fatores associados e preditores da qualidade de vida nesta corporação. Além disso, o delineamento transversal não permite inferências sobre causa-efeito.

\section{Conclusão}

A rede de correlações entre as dimensões do Modelo Desequilíbrio Esforço-Recompensa, resiliência e qualidade de vida em policiais militares indicou associação negativa entre esforço e excesso de comprometimento com os domínios físico e meio ambiente. O domínio meio ambiente foi associado positivamente à recompensa; $\mathrm{e}$ a resiliência, considerada um fator de proteção para a qualidade de vida nesta população, associou-se positivamente com os domínios geral, físico e psicológico. Constatou-se que o estresse psicossocial e a resiliência interferem na qualidade de vida dos policiais civis, uma vez que as dimensões esforço, recompensa e excesso de comprometimento do modelo DER e a resiliência se correlacionaram com todos os domínios do WHOQOL.

Trabalhadores como os policiais militares merecem maior atenção, uma vez que a atuação na segurança pública predispõe alto custo e baixo ganho, que reflete na capacidade produtiva do profissional e no serviço ofertado pelos mesmos. Profissionais de saúde têm competência para oferecer ações a favor da qualidade de vida e do rendimento laboral destes profissionais. Contudo, investimentos em infraestrutura e política de valorização da categoria são fundamentais para a saúde e segurança do grupo estudado. 


\section{Colaboradores}

JP Tavares contribuiu na concepção do projeto, coleta de dados, análise de dados, escrita do artigo, aprovação da versão publicada. LS Vieira, RF Ceccon e WL Machado contribuíram na análise dos dados, escrita do artigo e aprovação da versão publicada. D Dal Pai e SBC Souza cooperaram na concepção do projeto, escrita do artigo e aprovação da versão publicada.

\section{Agradecimentos}

Ao Conselho Nacional de Desenvolvimento Científico e Tecnológico (CNPq) e Pró-Reitoria de Pesquisa da UFRGS (PROPESQ) pelo apoio financeiro.

À professora doutora Liana Lautert pela participação da concepção e planejamento do projeto de pesquisa.

\section{Referências}

1. Galatzer-Levy IR, Steenkamp MM, Brown AD, Qian M, Inslicht S, Henn-Haase C, Otte C, Yehuda R, Neylan TC, Marmar CR . Cortisol response to an experimental stress paradigm prospectively predicts long-term distress and resilience trajectories in response to active police service. $J$ Psychiatr Res 2014; 56:36-42 [cited 2018 Sept 25]. Available from: https://www.sciencedirect.com/science/article/pii/ S0022395614001332?via\%3Dihub

2. Alexopoulos EC, Palatsidi V, Tigani X, Darviri C. Exploring Stress Levels, Job Satisfaction, and Quality of Life in a Sample of Police Officers in Greece. Saf Health Work 2014 [internet]; 5:210-215 [cited 2018 Sept 24]. Available from: https://www.sciencedirect.com/ science/article/pii/S2093791114000523?via\%3Dihub

3. Strahler J, Ziegert T. Psychobiological stress response to a stimulated school shooting in police officers. Psychoneuroendocrinology 2015; 51:80-91 [cited 2018 Sept 25]. Available from: https://www. sciencedirect. com/science/article/pii/S0306453014003564?via\%3Dihub

4. Souza ERD, Minayo MCDS, Silva JG, Pires TDO. Factors associated with psychological distress among military police in Rio de Janeiro, Brazil. Cad Saude Publica 2012; 28(7):1297-1311 [cited 2018 Sept 29]. Available from: http://www.scielo.br/scielo.php?script=sci_arttext\&pid=S0102-311X2012000700008\&lng=pt\&tlng $=$ pt.

5. Pelegrini A, Cardoso TE, Claumann GS, Pinto AA, Felden EPG. Perception of work conditions and occupational stress among civil and military police officers of special operations units. Cad Bras Ter Ocup 2018 26(2):423-430 [cited 2018 Sept 29]. Available from: http://www.cadernosdeterapiaocupacional.ufscar.br/ index.php/cadernos/article/view/1946

6. Mello-Silva ACC, Brasil VV, Minamisava R, Oliveira LMAC, Cordeiro JABL, Barbosa MA. Quality of life and psychological trauma in firearm violence victims. Texto Contexto Enferm 2012; 21(3):558-565 [cited 2019 Jan 19]. Available from: http://www.scielo.br/scielo.php?script=sci_arttext\&pid=S0104-07072012000300010\&lng=pt\&tlng=pt. http://dx.doi.org/10.1590/S0104-07072012000300010

7. Bartz ML, Quartieri ES, Menezes G. Criminalidade no Rio Grande do Sul: uma análise econométrica para os COREDES no ano de 2010. Rev Brasil Estud Regionais Urbanos 2018; 12(1):110-128 [cited 2019 Jan 18]. Available from: https://www.anpec.org.br/sul/2017/ submissao/files_I/i3-d787182219d9cad2acd9ea5e850a5d05.pdf

8. Siegrist J. Effort-Reward Imbalance Model in: Stress: Concepts, Cognition, Emotion, and Behavior, 2016. London: Academic Press Elsevier; 2016 [cited 2019 Jan 18]. Available from: https://www.sciencedirect. com/science/article/pii/B9780128009512000091?via\%3Dihub

9. Britt TW, Shen W, Sinclair RR, Grossman MR, Klieger DM. How much do we really know about employee resilience? Ind Organ Psychol 2016; 9(2):378-404 [cited 2018 Dec 13]. Available from: https://www. cambridge.org/core/journals/industrial-and-organizational-psychology/article/how-much-do-we-really-know-about-employee-resilience/0B87A5E241D67E14396E27CF82379FA3. 
10. The WHOQOL Group. The World Health Organization quality of life assessment (WHOQOL): position paper from the World Health Organization. Social Sci Med 1995; 10:1403-1409.

11. Organización Mundial de la Salud(OMS). Promoción de la salud: glosario. Genebra: OMS; 1998.

12. Meulen E, Velden PG, Setti I, Veldhoven MJPM. Predictive value of psychological resilience for mental health disturbances: A three-wave prospective study among police officers. Psych Research 2018; 260:486-494 [cited 2018 Dec 13]. Available from: https://www.sciencedirect.com/science/article/pii/ S0165178117312532?via\%3Dihub

13. Guimarães LAM, Mayer VM, Bueno HPV, Minari MRT, Martins LF. Burnout syndrome and quality of life in the military police and civilian. Rev Sul Americ Psicol 2014; 2(1):98-122 [cited 2018 Oct 25]. Available from: http://www.revista.unisal.br/am/index.php/ psico/article/view/32

14. Siegrist J, Wege N, Pühlhofer F, Wahrendorf M. A short generic measure of work stress in the era of globalization: effort-reward imbalance. Int Arch Occup Environ Health 2009; 82(8):1005-1013 [cited 2018 Sept 12]. Available from: http://link. springer.com/ article/10.1007/s00420-008-0384-3

15. Sá G, Farias S, Griep R, Portela L. Psychosocial Stress of nurses in oncology: Effort-Reward Imbalance Scale. Occup Environ Med 2014; 71(Supl.1):A61-A61 [cited 2018 Oct 10]. Available from: https://oem.bmj. com/content/71/Suppl_1/A61.1

16. Wagnild GM, Young HM. Development and psychometric evaluation of resilience scale. J Nurs Meas 1993; 1(2):165-78 [cited 2018 Sept 12]. Available from: https://www.ncbi.nlm.nih.gov/pubmed/7850498

17. Pesce RP, Assis SG, Avanci JQ, Santos NC, Malaquias JV, Carvalhaes R. Cross-cultural adaptation, reliability and validity of the resilience scale. Cad Saude Pública 2005; 2(21):436-448 abr [cited 2018 Oct 10]. Available from: http:// www.scielo.br/scielo.php?script=sci_arttext\&pi$\mathrm{d}=$ S0102-311X2005000200010\&lng $=\mathrm{pt} \& \mathrm{t} \operatorname{lng}=\mathrm{pt}$

18. Fleck MPA. The World Health Organization instrument to evaluate quality of life (WHOQOL-100): characteristics and perspectives. Cienc Saude Coletiva 2000; 5(1):33-38 [cited 2018 Oct 11]. Available from: http://www.scielo.br/scielo.php?script=sci_arttext\&pid=S1413-81232000000100004\&lng $=p t \& t \operatorname{lng}=p t$

19. Epskamp S, Fried EI. A tutorial on regularized partial correlation networks. Psychol Methods 2018; 23(4):617-634 [cited 2018 Dec 14]. Available from: https://psycnet.apa.org/doiLanding?doi=10.1037\%2Fmet0000167

20. Yang Z, Algesheimer R, Tessone CJ. A Comparative Analysis of Community Detection Algorithms on Artificial Networks. Scientific Reports 2016; 6:30750 [cited 2018 Dec 14]. Available from: https://www.nature. com/articles/srep30750.

21. Souza Filho MJ, Noce F, Andrade AGP, Calixto RM, Albuquerque MR, Costa VT. Evaluation of quality of life in military police officers. R Bras Ci Mov 2015; 23(4):159-169 [cited 2018 Nov 18]. Available from: http://www.bibliotekevirtual.org/index.php/201302-07-03-02-35/2013-02-07-03-03-11/1550-rbcm/ v23n04/17332-avaliacao-da-qualidade-de-vida-de -policiais-militares.html
22. Tesfaye T, Shikur B, Shimels T, Firdu N. Prevalence and factors associated with diabetes mellitus and impaired fasting glucose level among members of federal police commission residing in Addis Ababa, Ethiopia. BMC Endocrine Disorders 2016; 16:68 [cited $2018 \mathrm{Nov}$ 18]. Available from: https://bmcendocrdisord.biomedcentral.com/articles/10.1186/s12902-016-0150-6

23. Reis Neta ESA, Fernandes Filho J, Cortez ACL. Nível de atividade física e estado nutricional de policiais militares na cidade de Floriano-PI. Rev Kinesis 2016; 34(1):84-101 [cited 2018 Nov 18]. Available from: https://periodicos.ufsm.br/kinesis/article/view/22273

24. Brasil VP, Lourenção LG. Quality of life of military police officers from the inland of São Paulo state. Arq Cienc Saúde 2017; 24(1):81-85 [cited 2018 Nov 18]. Available from: http://www.cienciasdasaude.famerp. br/index.php/racs/article/view/511

25. Almeida DM, Lopes LFD, Costa VMF, Santos RCT. Job Satisfaction of the Rio Grande do Sul Military Policemen: a Quantitative Study. Psicol Cienc Prof 2016; 36(4):801-815 [cited 2018 Nov 23]. Available from: http://www.scielo.br/scielo.php?script=sci_arttext\&pid=S1414-98932016000400801\&lng=pt\&tlng=pt.

26. Andersen JP, Papazoglou k Nyman M, Koskelainen M, Gustafsberg H. Fostering resilience among police. Journal of Law Enforcement 2015; 5(1):1-11 [cited 2018 Nov 5] Available from: Available from: https:// tspace.library.utoronto.ca/handle/1807/73820

27. Lee Jong-Ku, HG Choi , Kim JY, Nam J, Kang HT, Koh SB, Oh SS. Self-resilience as a protective factor against development of post-traumatic stress disorder symptoms in police officers. Ann Occup Environ Med 2016; 28(1):58 [cited 2018 Sept 18]. Available from: https://www.ncbi.nlm.nih.gov/pmc/articles/ PMC5067890/

28. Silva FC, Hernandez SSS, Gonçalves E, Castro TLS, Arancibia BAV, Silva R. Quality of life of policemen: a systematic review of observational studies. Rev Cubana Med Mil 2014; 43(3):341-351 [cited 2018 Dec 2]. Available from: http://bvs.sld.cu/revistas/mil/ vol43_3_14/mil08314.htm

29. Suresh RS, Anantharaman RN, Angusamy A, Ganesan J. Sources of Job Stress in Police Work in a Developing Country. Int J Business Management 2013; 8(13) [cited 2018 Nov 22] Available from: http://www.ccsenet.org/journal/index.php/ijbm/article/view/26454

Artigo apresentado em 14/04/2019

Aprovado em 24/07/2019

Versão final apresentada em 26/07/2019

Editores-chefes: Romeu Gomes, Antônio Augusto Moura da Silva 